unemployment among scientists and engineers. The bill should, however, be seen more in terms of its impact on science policy in Washington, for it represents a fundamental realignment of policy making, by taking responsibility away from mission oriented agencies and placing it in the hands of what would in effect be a department of science and technology. If the bill does no more than stimulate debate on that issue, it will have done a great service to science policy.

\section{Wish list for the 70s}

by our Washington Correspondent ASTRONOMY, like many other branches of science in the United States, has been tightening its belt in the past few years. levelling of financial support, a lull in There has been the familiar pattern of a the funding of new projects and a rapidly increasing production of PhDs. And the situation has been particularly acute in radio astronomy, where virtually no new facilities have been given the go-ahead since the mid $1960 \mathrm{~s}$, but where there has been a rapid increase in new ideas and knowledge. But the situation, at least in some branches of astronomy, may be improving-the National Science Foundation has been given the green light for the very large array system of radio antennae (VLA), and a site for the project has already been chosen in New Mexico.

Nevertheless, according to a committee of the National Academy of Sciences, unless the budget for astronomy in the United States increases by at least 5.5 per cent a year in real terms,

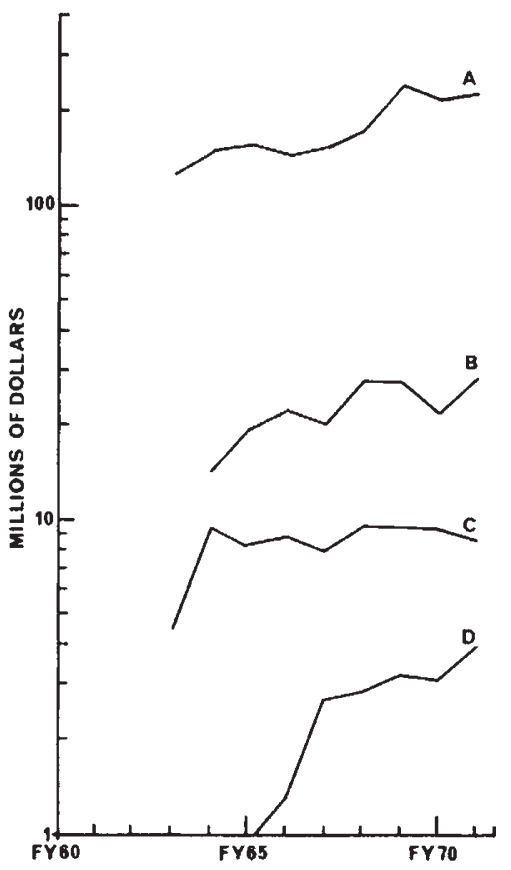

Fig. 1 Federal obligations for basic re search in astronomy. A, NASA: B, NSF, including major research instruments (as does NASA); C, Department of Defense; D, Smithsonian Institution. during the rest of the $1970 \mathrm{~s}$, astronomers will be hard done by. The committee, under the chairmanship of Dr Jesse L. Greenstein of California Institute of Technology, has drawn up a list of eleven projects which it suggests are vital for the health of astronomy in the US, and which would cost a total of $\$ 1,200$ million in construction and operating funds during the next decade. The wish list is the product of an exhaustive survey of US astronomy, conducted by eleven panels of scientists and drawn together by Dr Greenstein's committee. (Astronomy and Astrophysics for the 1970s, National Academy of Sciences, 2101 Constitution Avenue NW, Washington DC 20418.)

The committee argues for such a budget increase chiefly on the basis that the recent slowdown in funding increases has come at a time when there is a flood of discoveries and new opportunities in astronomy, and where the potential for space based research is rapidly expanding. The situation, the committee believes, "forebodes an imminent crisis in support per scientist trying to do research in astrophysics at a time when the quality, depth of education, and technological skills of those entering the profession are the highest that they have ever been".

At the top of the list of projects are four which the committee believes are essential for the future of astronomy in the United States. They are:

- the VLA, accompanied by a $\$ 25$ million increase in funding for medium sized university operated radio telescopes during the next ten years;

- a programme for upgrading all major US optical telescopes with electrooptical detectors and construction of a multimirror optical telescope equivalent to a conventional 150 or 200 inch instrument, followed by construction of a similar 400-600 inch telescope. The total in construction money for such optical facilities would be about $\$ 50$ million over the next decade.

- a doubling of expenditure on infrared astronomy, to be kicked off by a balloon survey of the sky for objects bright in the far infrared. Such a survey, the committee believes, could be conducted for less than $\$ 200,000$, and it suggests a total budget for an expanded infrared programme of $\$ 25$ million over the decade, compared with present expenditures of less than $\$ 2$ million a year;

- the final essential programme is the high energy astronomy programme that has already been given the go-ahead by NASA. The committee suggests that NASA should now seek authorization for pointable HEAOs to supersede the first rotating HEAO, and that four missions be flown in the 1970s.

These four projects together would take about half the $\$ 1,200$ million sug- gested by the committee as a reasonable budget for astronomy in the 1970s, but they should not be allowed to crowd out the seven other projects assigned a lower priority but which are nevertheless "essential to the health and balance of the total astronomical enterprise". These include construction of a very large millimetre wavelength antenna for interstellar spectroscopy and to study the early evolution of quasars; a doubling in support for aircraft, balloon and rocket based observations, particularly for ultraviolet studies to back up the Orbiting Astronomy Observatory series of spacecraft; increased support for theoretical astronomy, especially for computing facilities, and a programme of optical space astronomy leading to the Large Space Telescope (LST).

The committee also throws its weight behind those who are campaigning to save the Orbiting Solar Observatory series of spacecraft, arguing for continuation of the programme for another six satellites. Unfortunately, however, while the committee's report has been awaiting publication, NASA has already deferred funding on two of the three remaining satellites, and there is even some doubt about the remaining one.

If the committee's recommendations were adopted, they would run up a bill of about $\$ 355$ million a year over the next decade, compared with present expenditures of $\$ 275$ million. One shortcoming of the report is that it does not predict the likely scientific consequences if the budget for astronomy in the US were to grow more slowly than its recommendations suggest. To judge by past trends, that is more than a possibility.

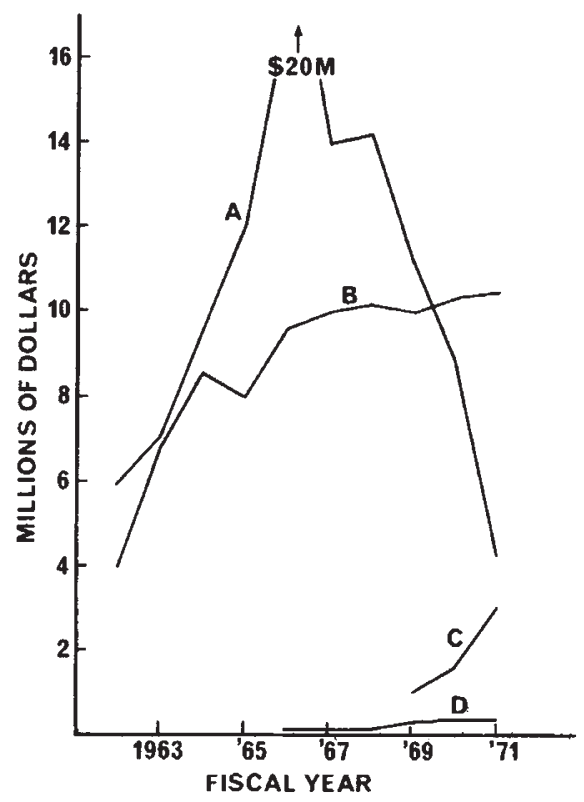

Fig. 2 NASA budgets for specific astronomy programmes which do not involve satellite observatories. A, Launch vehicles: $B$, sounding rockets; $C$, airplane observatory; $\mathrm{D}$, data analysis. 